\title{
CARACTERÍSTICAS DE LA ACTIVIDAD TURÍSTICA QUE UTILIZA AL LOBO MARINO DE CALIFORNIA COMO RECURSO NO EXTRACTIVO EN LA BAHÍA DE MAZATLÁN, SINALOA, MÉXICO
}

\section{CHARACTERISTICS OF THE TOURISM ACTIVITY THAT USES THE CALIFORNIA SEA LION AS A NON-EXTRACTIVE RESOURCE IN THE BAY OF MAZATLÁN, SINALOA, MEXICO}

Belén Díaz-Maestre*

Oscar Guzon-Zatarain * *

Sandra Pompa-Mansilla***

Mayra Ixchel Grano-Maldonado****

Blanca Roldán-Clarà *****

RESUMEN: En el noroeste de México el lobo marino de California, Zalophus californianus, es la única especie de mamífero marino (pinnípedo) residente en el Golfo de California y es considerado uno de los íconos turísticos del puerto de Mazatlán, Sinaloa. En la Bahía de Mazatlán existe una lobera temporal durante los meses de invierno y primavera en una pequeña roca llamada Roca Tortuga. La cercanía de la lobera permite a los operadores de servicios turísticos una interacción directa con los lobos marinos, siendo los paseos en bote una atracción, además la presencia de lobos marinos coincide con la temporada alta de visitación turística al puerto. El objetivo del estudio fue identificar el número de usuarios del sector turismoque utiliza al lobo marino como atractivo en sus paseos por la Bahía de Mazatlán y determinar la derrama económica. Para obtener la información se realizaron 55 encuestas y 52 entrevistas semi-estructuradas a operadores turísticos en el período de noviembre de 2011

Recibido 05 de abril 2020/Aceptado 05 de marzo 2021

* Maestra en Ciencias por la Universidad Autónoma de Sinaloa.

**Maestro en Ciencias por el CICESE, Director General de Onca Exploraciones S.A. de C.V.

https: / / orcid.org/0000-0002-7620-9885

***Doctora en Ecología, UNAM. Directora de Fuego Verde S.C. https://orcid.org/0000-000 1-9627-6826

**** Doctora en Filosofía de la Ciencia, Universidad Autónoma de Occidente. https:/ / orcid.org/0000-000 1-75 19-379X

$* * * * *$ Doctora por la Universidad Autónoma de Baja California. Universidad Autónoma de Occidente.

blancamar@gmail.com https://orcid.org/0000-0002-6091-4256 
a mayo de 2012. Los resultados de esta investigación muestran que 12 operadores turísticos, empleando 18 embarcaciones, ofrecieron a los lobos marinos de Roca Tortuga como atractivo turístico en sus recorridos a 35.531 visitantes. La derrama económica generada en esta actividad a lo largo del período analizado fue de $\$ 11.425 .120$ pesos mexicanos (MXN), de los cuales \$3.714.237.00 (MXN) equivalentes al 30\%, corresponden a visitas a Roca Tortuga exclusivamente para avistar lobos marinos. Esta actividad podría representar una alternativa sostenible para el uso de los recursos naturales con un beneficio socioeconómico para los locales y como una actividad con un gran potencial para el desarrollo de actividades de educación para la conservación del ecosistema.

Palabras clave: Zalophuscalifornianus, Lobo marino de California, Mazatlán, Ecoturismo, actividad turística, México.

ABSTRACT: In northwestern Mexico, the California sea lion Zalophuscalifornianus is the only species of marine mammal (pinniped) resident in the Gulf of California and is considered one of the touristic icons of the port of Mazatlán, Sinaloa. In Mazatlan Bay, there is a temporary sea lion rookery during the winter and spring months on a small rock called Roca Tortuga (Turtle Rock). The proximity of the sea lion trap allows tourist service operators to interact directly with the sea lions, and boat rides are an attraction; in addition, the presence of sea lions coincides with the high season of tourist visitation to the port. The objective of the study was to identify the number of users of the tourism sector that use the sea lion as an attraction in their tours of Mazatlan Bay and to determine the economic spill. To obtain the information, 55 surveys and 52 semi-structured interviews were conducted with tour operators from November 2011 to May 2012. The results of this research show that 12 tour operators, using 18 boats, offered Roca Tortuga sea lions as a tourist attraction on their tours to 35.531 visitors. The economic income generated by this activity during the period analyzed was \$1 1.425.120 MXN, of which \$3.714.237 MXN (30\%) corresponds to visits to Roca Tortuga exclusively to see sea lions. This activity could represent a sustainable alternative for the use of natural resources with a socioeconomic benefit for the locals and as an activity with great potential for the development of educational activities for the conservation of the ecosystem.

Keywords: Zalophus californianus, California sea lion, Mazatlán, Ecotourism, tourist activity, Mexico. 


\section{INTRODUCCIÓN}

El ecoturismo puede definirse como cualquier actividad recreativa que implique el avistamiento de seres vivos en su medio natural y que incorpore alguna forma de educación ambiental, tiene un bajo nivel impacto, pretende ser sostenible $y$, en algunos casos, apoya los esfuerzos locales de conservación. Además, el ecoturismo es una de las industrias de más rápido crecimiento a nivel mundial Uohnson et al., 20191. Una serie de estudios han demostrado la relevancia de la satisfacción de los turistas y la gestión sostenible de las actividades relacionadas con la naturaleza (Das y Chetteriee, 2015; Secretaría de Turismo [SECTUR], 2007; López-Espinosa, 2002). El ecoturismo marino se ha desarrollado recientemente, comparado con otras formas de ecoturismo (Miller 1993). Sin embargo, el ecoturismo marino, relacionado con actividades como avistamiento de ballenas y tiburones, snorkel, el buceo y la pesca recreativa, atraen 120 millones de participantes por año a nivel mundial, generando más de 50 mil millones de dólares por año y apoyando más de un millón de puestos de trabajo (Cisneros-Montemayor y Sumaila, 2010). En Latinoamérica, los estudios acerca del desarrollo e impactos del ecoturismo van al alza y son sujeto importante de investigación desde puntos de vista sociales, económicos, ambientales y culturales (Wondirad, 2019). En el caso de México, el ecoturismo marino enfocado en especies como ballenas, lobos marinos, tiburones y experiencias de buceo y snorkel, se encuentra en pleno desarrollo e cor investigación (Garrod y Wilson, 2003). Existen estudios relacionados al ecoturismo y conservación de lobos marinos en Latinoamérica (Bechtold y Parisi, 2020;
Mathis y Rose, 20161, más éste es el primer trabajo que evalúa los efectos del turista y el lobo marino en estas comunidades en la bahía de Mazatlán, Sinaloa, México.

En la actualidad, el turismo es un sector fundamental en la economía global. Para garantizar que el turismo contribuya de manera sostenible a la economía regional, es esencial preservar las condiciones ambientales, brindar oportunidades para que los turistas experimenten la naturaleza y aprendan sobre la importancia de la conservación de la biodiversidad, teniendo en consideración las necesidades de los visitantes (Gallagher y Hammerschlag, 2011 ; Drum y Moore, 2004). En el caso de Sinaloa, Mazatlán es considerado uno de los destinos turísticos de sol y playa más importantes de este país, por su ubicación privilegiada, el clima y la geografía de la bahía y su zona marina. En este sentido, el Consejo para el Desarrollo Económico de Sinaloa (CODESIN) define que la oferta turística de Mazatlán contempla actividades derivadas del paisaje que determinan los atractivos del Puerto. Mazatlán ofrece un gran potencial para la integración de estos recursos y los productos turísticos que permitan aprovechar de manera sustentable la gran riqueza ecológica (SECTUR, 2014). Mazatlán es la segunda ciudad más importante de Sinaloa, al contar con una economía mayormente terciaria, con el $66 \%$ de la población dedicada al comercio y servicios, principalmente al turismo, sector que generó el 67\% de las remuneraciones totales en 2016 (Instituto de Estadística en América Latina y el Caribe [INEGI], 2020). Es por esto que se considera una ciudad con vocación económica ligada al turismo, actividad que genera el 47\% del PIB del municipio. Mazatlán se encuentra entre los doce principales centros turísticos del país por actividad hotelera, sin embargo, no se 
considera un destino turístico con alto nivelde gasto /Comisión Intersecretarial para el Manejo Sustentable de Mares y Costas de México [CIMARES], 20121. A pesar de sus características turísticas, es importante mencionar el vertiginoso ascenso en el turismo que visita Sinaloa (4.7 millones de personas en 2019,20\% más que en 2018 ) (Toledo, 2020) y que el 70\% de éste se concentra en Mazatlán. De la mano con la visita de turistas, el crecimiento en infraestructura turística sin planeación a largo plazo y carente de criterios de sostenibilidad (Rodríguez y Valiente, 2019) amenazan la conservación de sus ecosistemas.

En este contexto, hacer converger los esfuerzos de conservación de la biodiversidad y el desarrollo del turismo ha dado lugar a escenarios ecoturísticos a nivel mundial (Habibullah et al., 2016). Así, el turismo de naturaleza o ecoturismo emerge como un contrapunto al turismo convencional y se enfoca en la promoción de lugares con gran capital natural. Estos objetivos fueron desarrollados en el concepto de ecoturismo que surge como resultado de la integración del desarrollo sostenible y el turismo y se centra en tratar de encontrar y fomentar alternativas para viajar con el objetivo de preservar y respetar el medio ambiente (Bringas y Ojeda, 2003). Los destinos ecoturísticos en Mazatlán, como producto, compiten en los ámbitos nacional e internacional. El puerto sinaloense ofrece una experiencia que el turista vive en el destino. En este contexto, el espacio geográfico que se instituye como destino turístico crea la experiencia turística la cual es una vivencia auténtica y única (Nava e lbarra, 2014; SECTUR, 2014).

Además de la experiencia turística vinculada a la naturaleza, esta actividad puede llevarse a cabo en Áreas Naturales Protegidas, sitios donde la biodiversidad a nivel mundial es identificada como única e irremplazable /Chung et al., 2018; Crofts, 20191 y donde participan las comunidades locales en los esfuerzos de conservación (Corrigan et al., 2018). Mazatlán presenta varias opciones de visitas en entornos naturales considerados como Áreas Naturales Protegidas (ANP) del orden federal, como las Islas Lobos, Venados y Pájaros frente a la bahía, todas ellas visibles desde el malecón, que forman parte del Área de Protección de Flora y Fauna (APFF) denominada "Islas del Golfo de California, Sinaloa" (Gobierno del Estado de Sinaloa, 1991 ). En dicho escrito oficial, las tres islas fueron decretadas como zona de reserva ecológica y zona de refugio de aves marinas migratorias, flora y fauna silvestre (Secretaría de Medio Ambiente, Recursos Naturales y Pesca [SEMARNAP], 20001. Entre otros entornos naturales protegidos se incluyen los islotes "Dos Hermanos y la Roca Tortuga", los cuales forman parte de una reserva ecológica estatal (Guzón-Zatarain et al., 2013). Dentro de esta reserva, se ofrece una oferta ecoturística que incorpora diversas actividades acuáticas como: paseos en kayak, tours a la isla venados, isla de la piedra y observación de ballenas, entre otros, y los distribuidores ecoturísticos como los tour operadores de lanchas o embarcaciones tienen el recurso acuático considerado como muy atractivo, dentro de la oferta turística es la obligada visita a los lobos marinos en la roca tortuga (SECTUR, $2014)$.

Algunas de las actividades más comunes del turismo náutico en el puerto incluyen paseos en lancha, deportes acuáticos como el buceo libre o snorkel, buceo SCUBA, kayak, motos acuáticas, bananas, pesca deportiva y, más recientemente, la observación 
de fauna silvestre como aves, ballenas, Los tours de observación de ballenas, delfines y las visitas a Roca Tortuga (RT) para delfines y snorkel en islas que realiza ver los lobos marinos. Actualmente, existen "Onca Exploraciones" son los únicos flotas de catamaranes y embarcaciones servicios orientados de manera exclusiva a menores que ofrecen diversos tipos de paseos, la observación de la vida silvestre con un tales como la visita a RT para observar a los importante componente educativo y lobos marinos (Figura 1). Sin embargo, la académico. Otras embarcaciones que mayoría no se realizan exclusivamente con visitan la RT para observar los lobos este fin, ya que también el paseo incluye una marinos son las que ofertan paseos de visita a la bahía, el tradicional recorrido por la pesca deportiva, ya sea de altura o de "jungla" (paseo por los manglares del Estero fondo, casi siempre a su regreso hacen de Urías). Estos servicios incluyen alimentos, una visita a RT para complementar sus bebidas y deportes acuáticos en las playas de servicios e incrementar la satisfacción de la Isla de la Piedra. Los únicos paseos que se sus clientes. También existen importantes realizan casi exclusivamente para ver a los visitantes provenientes de embarcaciones lobos marinos y que también son los más privadas y otro tipo de vehículos acuáticos cortos en duración así como los más como kayak y jet skies.

económicos, son los ofrecidos en embarcaciones menores, pertenecientes a las Los lobos marinos de California (Zalophus cooperativas de los embarcaderos de las californianus) son mamíferos acuáticos, zonas conocidas como Playa Sur y de la Isla única especie de pinípedo en Sinaloa, de la Piedra Unión de Propietarios de considerados como uno de los íconos más Canoas, Botes y Lanchas CROM y Unión de importantes de la biodiversidad marina en Propietarios de Lanchas de la Isla de la Piedra México. En Mazatlán estos animales A.C.I, así como algunos que parten desde las poseen un monumento colocado flotas cercanas al faro en el puerto principal. estratégicamente para señalar la cercanía Recientemente, también los pescadores del con el acuario, otro sitio de interés turístico sitio Playa Norte han ofertado paseos en (SECTUR, 2014). Estos animales son de lancha, los cuales tienen, durante la gran relevancia local y catalogados como temporada, la finalidad de observar a los "Visitantes distinguidos" (Camacho, lobos marinos. 2016).

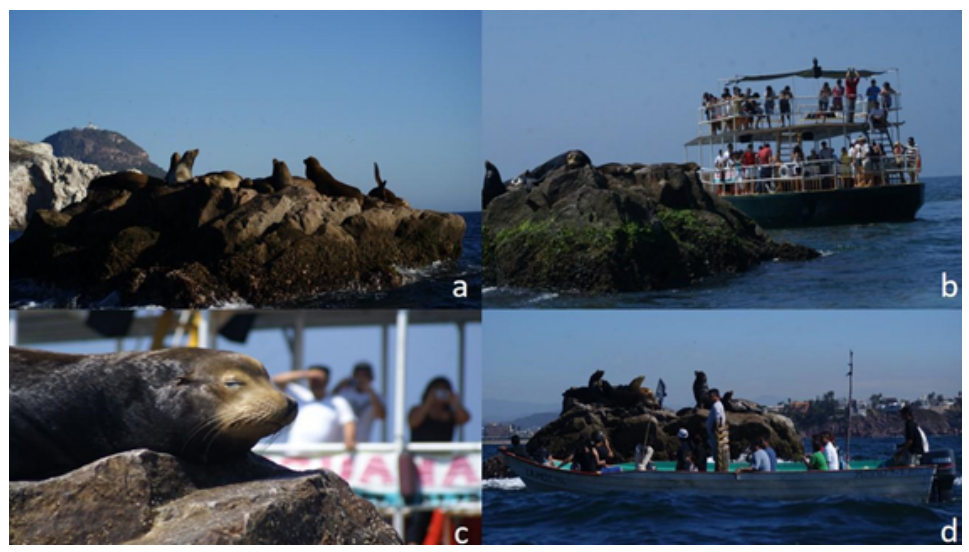

Figura 1. Roca Tortuga (RT), a) colonia temporal de lobo marino Zalophus californianus en Mazatlán, Sinaloa. b-d) embarcaciones turísticas que visitan a los lobos marinos como recurso no extractivo en RT. Fuente: Acervo fotográfico de Onca Exploraciones S.A. de C.V. 
Los lobos marinos en vida libre se especie. Sin embargo, también se podría distribuyen en el norte del Océano considerar como una plataforma de Pacífico, desde Columbia Británica, oportunidad para educar a miles de Canadá, hasta la región central de turistas -foráneos y locales-. Ahora bien, México, incluyendo el Golfo de California la población invernal del lobo marino ha (King, 1983). El lobo marino se encuentra sido investigada escasamente por la protegidos en la categoría de Sujeta a comunidad científica, desconociéndose protección especial ( $\mathrm{Pr}$ ) en la Norma aspectos no solo biológicos, sino también Oficial Mexicana económicos y sociales entorno al efecto en NOM-059-SEMARNAT-2010.

Este el turismo, por lo que los resultados de este pinnípedo es altamente gregario, estudio resultan relevantes para conocer la agrupándose en islas, islotes y sitios interacción de esta población con las protegidos de depredadores, formando actividades productivas invernales en este loberas (Peterson y Bartholomew, 1967). puerto.

Durante los viajes exploratorios para alimentarse lejos de sus zonas reproductivas, los sitios de descanso son ocupados principalmente por juveniles y machos sub-adultos y adultos, por lo que algunos lobos marinos se encuentran en Mazatlán, específicamente en el islote protegido de Roca Tortuga (RT) que puede albergar a más de 30 individuos (Aurioles-Gamboa y Zavala-González, 1994).

El espacio de congregación temporal de los lobos se encuentra al sur de la bahía de Mazatlán y se le conoce como Roca Tortuga (RT) y representa la ubicación más sureña de México (Figura 2), siendo un sitio de descanso para la recuperación de los individuos después de viajes intensivos de forrajeo (alimentación), interacción con otros individuos, evasión de depredadores, proceso de muda de pelo y viajes migratorios. Es muy importante señalar que RT es visitada por miles de turistas al año (Diaz-Maestre, 2017), representa así un importante atractivo turístico y se ha convertido en una oferta obligada de los prestadores de servicios turísticos en la bahía, convirtiéndose en una actividad que si no se hace correctamente, puede vulnerar a la

\section{DESARROLLO DEL TEMA ANTECEDENTES}

En 2012, preocupados por la falta de conocimiento sobre la sustentabilidad de la actividad turística en Mazatlán, Costeros Sustentables A.C. (ECOS) impulsó un proceso de planeación de turismo sustentable para la bahía de Mazatán en coordinación con la dirección del APFF, Islas del Golfo de California Sinaloa, de la Comisión Nacional de Áreas Naturales Protegidas (CONANP) y con el apoyo financiero del Fondo Mexicano para la Conservación de la Naturaleza, A.C. (FMCN). Se hicieron una serie de talleres en los que se convocó a los diferentes actores del sector turístico de Mazatlán a sumarse con sus capacidades al desarrollo de un proceso adaptativo, incluyente y participativo para 
planear el desarrollo sustentable de la actividad turística, y se desarrolló una visión común de turismo sustentable para la bahía de Mazatlán al 2025. Se realizó un diagnóstico del estado actual de la sustentabilidad de la actividad turística, en el cual se calificó el desempeño de los prestadores de servicios turísticos de Mazatlán en relación a los Criterios Globales de Turismo Sustentable (GSTC, por sus siglas en inglés), mismos que están avalados por la Organización Mundial del Turismo de las Naciones Unidas y han sido adoptados por SECTUR Federal como los lineamientos básicos para el aprovechamiento sustentable de los recursos naturales y culturales del país. Los resultados de este diagnóstico indicaron que la sustentabilidad de la actividad turística en Mazatlán se encontraba en un estado de "atención preventiva", con una calificación en semáforo amarillo (Guzón-Zatarain et al., 2013). Se sugirió que las empresas turísticas refuercen su compromiso con referencia a la conservación de la biodiversidad y realicen mejores esfuerzos para contribuir a salvaguardar los sistemas que soportan la vida en la región. Para lograrlo, es fundamental que la comunidad local logre desarrollar un sentido legítimo de propiedad sobre su patrimonio histórico, cultural y natural, para que los ciudadanos se sientan orgullosos de su territorio y se conviertan en mejores anfitriones del destino. Además, se deberían establecer sinergias institucionales encaminadas a la protección, conservación y aprovechamiento responsable de los recursos naturales. Por otro lado, las fortalezas del sector sugieren que la implementación del modelo de turismo sustentable en Mazatlán no sólo es viable, sino una necesidad parapoder competir en igualdad de condiciones con otros destinos y aprovechar las oportunidades que representan las nuevas tendencias en los mercados turísticos actuales (Guzón-Zatarain et al., 20131 .
Paralelamente se llevó a cabo la iniciativa "Hacia un modelo de turismo competitivo y sustentable en Mazatlán y el sur de Sinaloa". Este proyecto aterrizado a nivel destino, se enfocó particularmente en las tendencias de desarrollo turístico costero, inversiones y financiamientos para su realización. Considerando implicaciones macroeconómicas, el proyecto estableció dos estrategias: 1) posicionar a Mazatlán como un destino turístico de sol y playa, cultural y sustentable y 2) definir criterios de competitividad y sustentabilidad que guíen a los nuevos desarrollos turísticos costeros del sur de Sinaloa (Espinosa Martínez, 2014).

\section{ÁREA DE ESTUDIO}

La Roca Tortuga (RT) se localiza al sur de la bahía de Mazatlán, a $3 \mathrm{~km}$ de distancia de la entrada del puerto comercial de Mazatlán (Figura 1 y 2) y a 9 km de distancia de la boca de la Marina Mazatlán, en las coordenadas geográficas $\quad 23^{\circ} 11^{1} 13,15^{\prime \prime} \mathrm{N} \quad y$ $106^{\circ} 26^{\prime} 45,68^{\prime \prime} O$. Este islote mide aproximadamente $30 \mathrm{~m} 2$. Con referencia a la biodiversidad reportada en la bahía de Mazatlán, en la zona intermareal de las islas e islote habita una gran diversidad de flora y fauna marina (Cifuentes-Lemus y Gaxiola-López, 2003).

\section{MÉTODO DE ESTUDIO}

Para caracterizar la actividad turística que utiliza al lobo marino como recurso en Mazatlán, se identificaron los usuarios que emplean al lobo como atractivo en sus paseos por la Bahía de Mazatlán. Se solicitó mediante oficio formal a Capitanía 


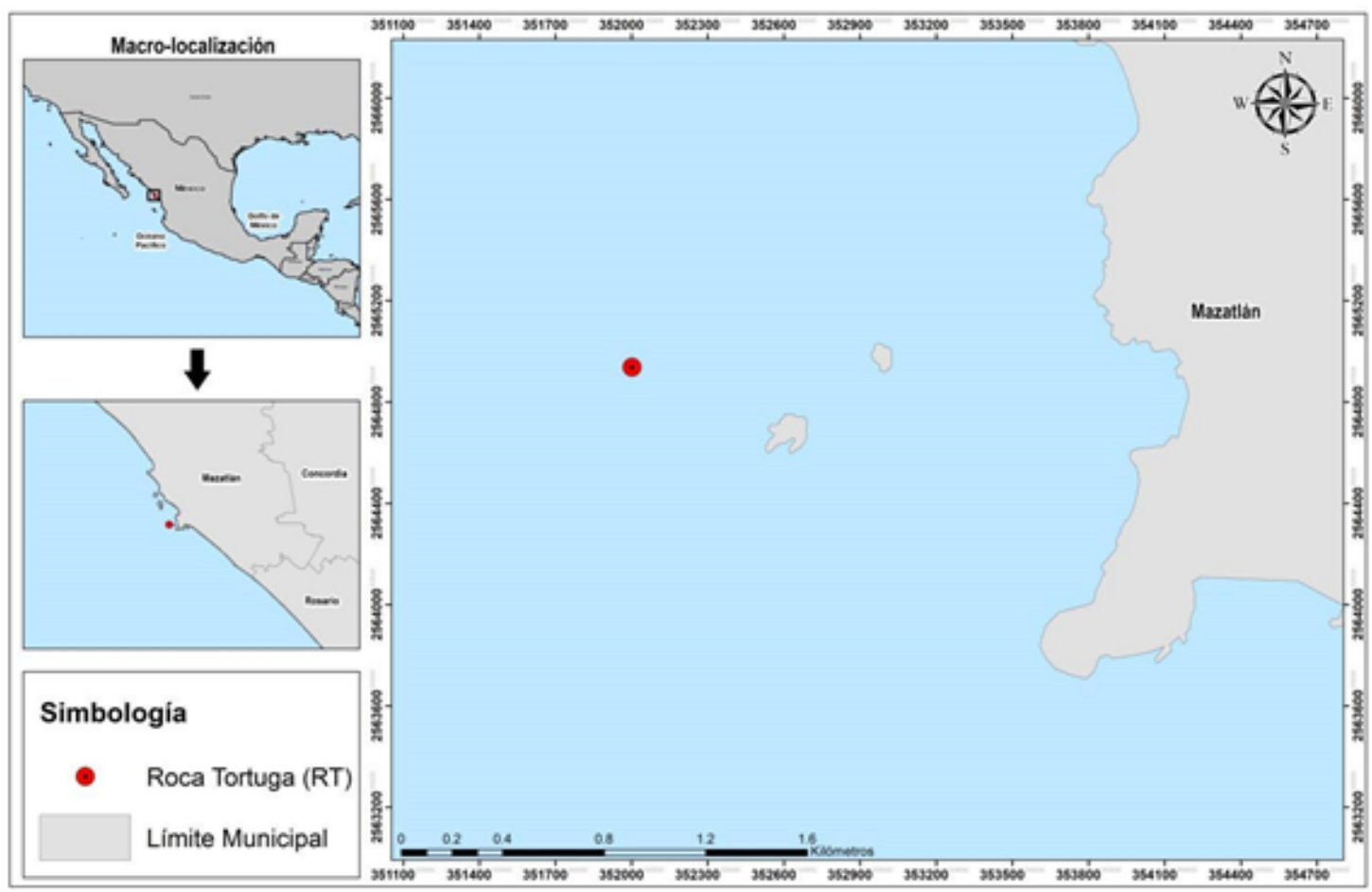

Figura 2. Localización de Roca Tortuga (RT) en la bahía de Mazatlán, Sinaloa, México. Elaborado por J. Yared Cortina A. (UTM 2564954.7 N 352003.4 E Zona 13N)

de Puerto información sobre los operadores con permiso turístico para navegar en la bahía.

Al mismo tiempo, se diseñó y aplicó una encuesta de 39 reactivos, siguiendo las características de un cuestionario con preguntas cerradas (Martín, 2011). El instrumento se estructuró en cuatro secciones (Díaz-Maestre et al., 2020) y los reactivos se calificaron considerando las frecuencias de las respuestas correctas para cada una de ellas. La encuesta fue auto administrada a 55 participantes (se proporcionó el cuestionario directamente a los respondientes), quienes la contestaron por si mismos marcando las respuestas con la ayuda de un grupo de voluntarios (Sampieri-Hernández et al., 2010). Durante la aplicación del cuestionario se consideró la observación participante (Aguiar, 2015; Angrosino, 20121 como una estrategia para recabar información de forma indirecta. La información obtenida fue transcrita a una base de datos en Excel y analizada acorde a las secciones del cuestionario. Además, se sostuvieron entrevistas semi-estructuradas (Trindade, 2016; Díaz-Bravo et al, 2013 ; Sierra, 19981, cara a cara, con 52 operadores que administran los servicios para determinar las características de su servicio, como precios, recorrido y tiempo del recorrido, entre otros datos.

Con base en datos de despacho de la Capitanía de Puerto, se estimó la derrama económica mínima siguiendo a Andersson (2001) y Kido y Cuéllar (2010) que genera la actividad turística en torno a los lobos marinos en la bahía de Mazatlán. Utilizando el número de pasajeros a bordo de viajes de turismo náutico y el precio promedio de venta al público, se estimaron los beneficios económicos, en términos de ingresos totales (moneda nacional, pesos 
mexicanos), generados por la venta de paseos de turismo náutico en la bahía de Mazatlán durante la temporada invernal 2010-2011. Los precios de venta al público se obtuvieron mediante comunicaciones telefónicas y entrevistas personales con los operadores correspondientes y se verificaron en distintas páginas de internet. Se consideró solo la información correspondiente a las embarcaciones de operadores que incluyen regularmente en su oferta de servicios la visita a RT para la observación de los lobos marinos.

\section{RESULTADOS \\ CARACTERIZACIÓN DE LA ACTIVIDAD TURÍSTICA}

El número total de embarcaciones que operaron durante la temporada invernal 201 1-2012, de las cuales se tiene registro $(n=105)$, coincidió con la información oficial de la Dirección General de Marina Mercante y la Dirección de Registro y Programas, sobre el número de permisos para la prestación de servicios de turismo náutico otorgados por la Capitanía de Puerto de Mazatlán vigentes durante el periodo 2003-2005. Según los registros de despacho proporcionados por Capitanía de Puerto para este estudio, entre el 3 de noviembre del 2011 y el 14 de mayo de 2012 (156 días), operaron un total de 105 embarcaciones transportando a 40.126 personas. Del total de embarcaciones, 62 operaron desde las flotas del puerto principal, mientras que 43 lo hicieron desde Marina Mazatlán. Las embarcaciones que operaron desde distintos embarcaderos del puerto principal le.g. Faro, Isla de la Piedra) incluyen 14 catamaranes grandes de pasajeros, 27 yates de pesca deportiva, cuatro pangas, una embarcación privada de investigación y una embarcación no identificada. Las embarcaciones que operaron desde Marina Mazatlán incluyen: 23 yates de pesca deportiva, 16 pangas de pesca deportiva y cuatro trimaranes de pasajeros. En la figura 3 se puede apreciar que el número de personas que contrataron servicios de turismo náutico aumentó progresivamente durante la temporada invernal desde $~ 1.000$ personas en noviembre de 2011 hasta rebasar las 5.000 personas en marzo de 2012 y un drástico aumento en abril, llegando casi a las 20.000 personas.

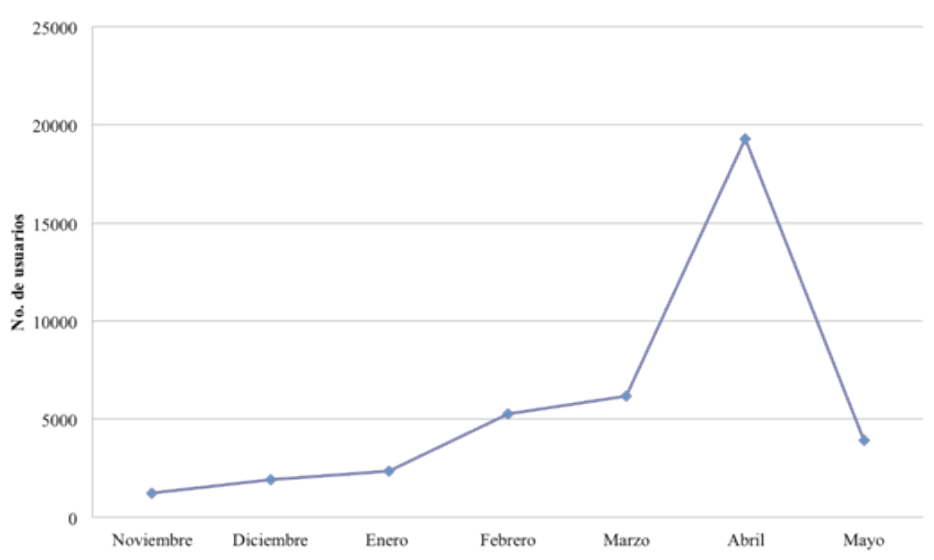

Figura 3. . Número de usuarios de servicios de turismo náutico en la bahía de Mazatlán durante la temporada invernal 201 1-2012 (3 de noviembre de 2011 al 14 de mayo de 2012). Fuente: Elaboración propia con datos de despachos de Capitanía de Puerto Mazatlán. 
Del total de usuarios de servicios de turismo diversas para paseos por la bahía, mientras náutico en la bahía de Mazatán para la que un 14\% contrató servicios para visitar la temporada invernal 2011-2012, para los Isla de la Piedra, los cuales también cuales se tenía información del tipo de incluyen un recorrido por la bahía y solo un servicio ofrecido $(n=29,418)$, la gran $8 \%$ contrató servicios de pesca deportiva mayoría (78\%) contrató embarcaciones (Figura 4).

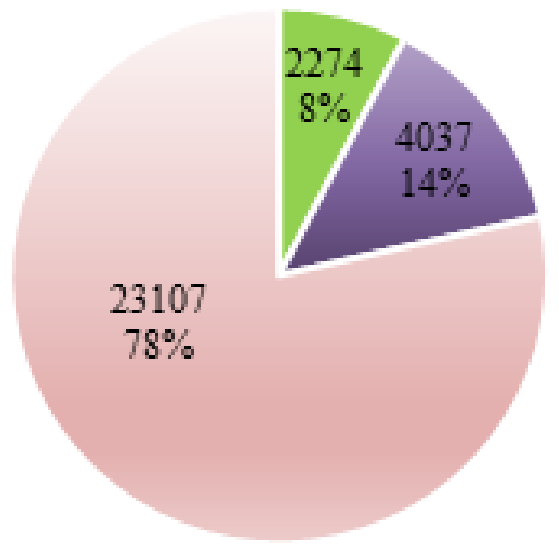

\section{- Pesca deportiva (yates/pangas) \\ - Isla de la Piedra (Catamaranes/pangas)}

\section{- Paseo por la bahía (Catamaranes/trimaran \\ es)}

Figura 4. Total de usuarios de servicios de turismo náutico en la bahía de Mazatlán por categoría de servicio durante la temporada invernal 2011 1-2012.

Fuente: Elaboración propia con datos de despacho de Capitanía de Puerto Mazatlán.

Un total de 18 embarcaciones y 12 que van desde los 48 hasta los 444 operadores turísticos realizan paseos por la pasajeros. Sin embargo, el promedio de bahía de Mazatlán con potencial de visita pasajeros por salida se encuentra entre 13 a RT para la observación de lobos marinos, y 24 para los catamaranes medianos que incluyendo aquellas que programan visitas operan desde Marina Mazatlán y desde a la Isla de la Piedra y a la Isla Venados. 16 hasta 72 pasajeros para la flota 14 embarcaciones (nueve operadores) decatamaranes que operan desde el puerto operan desde las flotas del puerto principal principal. Las embarcaciones de mayor y otras cuatro (tres operadores) desde la Marina Mazatlán. Considerando los viajes realizados por estas embarcaciones, se estimó que el total de usuarios que visitaron RT para la observación de lobos marinos durante la temporada invernal 2011-2012 fue de 35.531 personas. Estas 18 embarcaciones -la mayoría catamaranes medianos y grandes, así como un barco camaronero modificado para turismo /Yate Fiesta)- cuentan con capacidades máximas capacidad como El Americano, El Titanik, El Costeño y el Yate Fiesta han sido observadas en ocasiones con más de 200 pasajeros a bordo. En la Figura 5 se puede apreciar que las ganancias del turismo náutico, particularmente de paseos por la bahía de Mazatlán, no es equitativa, ya que tan solo dos operadores (cuatro catamaranes: Americano, Titanik y Puesta de Sol I yll) obtienen más de la mitad de los usuarios que contratan estos servicios. 


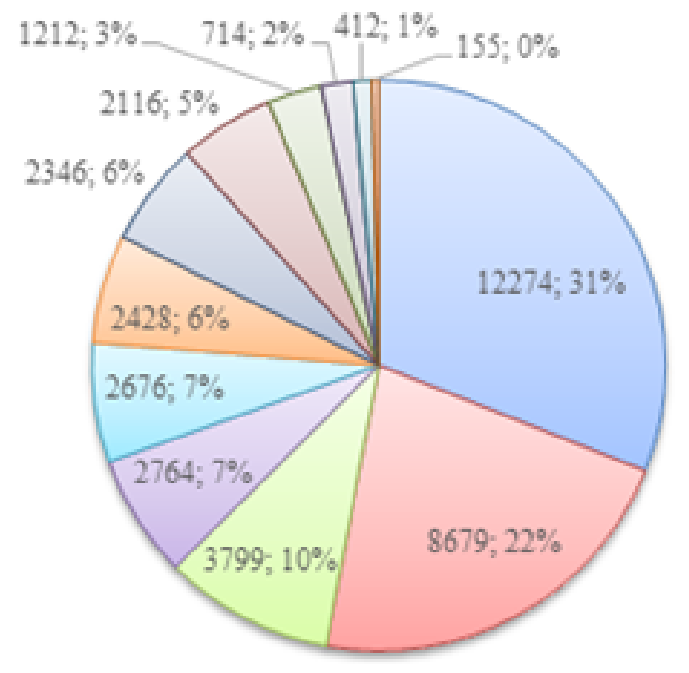

口Puesta del Sol I y II

口E1 Amerikano y Titanik

$\square \mathrm{E} 1$ Costeño

口Playa Azul

口Konakai y Kolonahe

$\square$ Playa Sur II

口Sábalo, III y IV

口Yate Fiesta y Vagabundo

口Iguana no.1

口isla de la Piedra

口Bula I

$\square$ Romance

Figura 5. Número total de usuarios por embarcación que contrataron servicios de paseos por la bahía de Mazatlán con potencial de visita a RT para la observación de lobos marinos durante la temporada invernal 2010-2011.

Fuente: Elaboración propia con datos de despacho de Capitanía de Puerto Mazatlán.

Se pueden distinguir dos segmentos del servicio de turismo náutico en el puerto, los que operan desde diferentes embarcaderos en el puerto principal y los que operan desde la Marina Mazatlán. En general, los servicios ofertados desde los embarcaderos cercanos a faro son más baratos y, por lo tanto, atraen a un turismo masivo y popular, mientras que los que operan desde Marina Mazatlán van dirigidos a un mercado con un mayor poder adquisitivo, por lo que son más costosos y a su vez de mayor calidad.

Derrama económica de la actividad turística en torno al lobo marino de California en la bahía de Mazatlán.
Como se había comentado anteriormente se calculó que el número de usuarios que contrataron servicios de paseos por la bahía con potencial de visita a RT para la observación de lobos marinos fue de 35.531 personas, lo que representa el $88,5 \%$ del total de embarcaciones $(n=40,126)$. Considerando los precios al público de los diversos servicios contratados por estos usuarios, se estimó que la derrama económica generada por la actividad turística en torno al lobo marino de California en Mazatlán fue de al menos de \$1 1.425.120 MXN para la temporada invernal 2011-2012 (Tabla 1). 


\begin{tabular}{|c|c|c|c|c|c|c|}
\hline $\begin{array}{l}\text { Nombre de la } \\
\text { embarcación }\end{array}$ & $\begin{array}{l}\text { Capacidad } \\
\text { máxima } \\
\text { pasajeros }\end{array}$ & $\begin{array}{l}\text { Pasajeros } x \\
\text { temporada } \\
\text { (2011-2012) }\end{array}$ & $\begin{array}{l}N^{\circ} \text { de } \\
\text { salidas }\end{array}$ & $\begin{array}{l}\text { Promedio } \\
\text { Pasajeros } \\
\text { x salida }\end{array}$ & $\begin{array}{c}\text { Precio al } \\
\text { público por } \\
\text { persona (MXN) }\end{array}$ & $\begin{array}{l}\text { Ingresos totales } \\
\text { (MXN) }\end{array}$ \\
\hline El Costeño & 160 & 4.064 & 72 & 56 & $\$ 250$ & $\$ 1.016 .000$ \\
\hline El Americano & 196 & 6.684 & 96 & 72 & $\$ 400$ & $\$ 2.673 .600$ \\
\hline Titanik & 444 & 2.160 & 54 & 40 & $\$ 400$ & $\$ 864.000$ \\
\hline Iguana & 90 & 1.157 & 21 & 55 & $\$ 200$ & $\$ 231.400$ \\
\hline Isla de la Piedra & 48 & 747 & 48 & 16 & $\$ 250$ & $\$ 186.750$ \\
\hline Playa Sur & 50 & 2.615 & 88 & 30 & $\$ 245$ & $\$ 640.675$ \\
\hline Playa Sur II & 75 & 2.504 & 53 & 47 & $\$ 200$ & $\$ 500.800$ \\
\hline $\begin{array}{l}\text { Puesta del Sol I y } \\
\text { ॥ }\end{array}$ & 90 & 8.323 & 137 & 61 & $\$ 300$ & $\$ 2.496 .900$ \\
\hline Sábalo I. III y IV & 75 & 2.333 & 80 & 30 & $\$ 225$ & $\$ 524.925$ \\
\hline Vagabundo & 50 & 118 & 7 & 17 & $\$ 300$ & $\$ 35.400$ \\
\hline Yate Fiesta & 195 & 2.016 & 54 & 37 & $\$ 245$ & $\$ 493.920$ \\
\hline Romance & 48 & 155 & 10 & 15 & $\begin{array}{l}\$ 3.500 \\
\text { (por salida) }\end{array}$ & $\$ 35.000$ \\
\hline Bula I & 48 & 400 & 31 & 13 & $\$ 650$ & $\$ 260.000$ \\
\hline Konakai & 48 & 1.038 & 55 & 19 & $\$ 650$ & $\$ 674.700$ \\
\hline Kolonahe & 48 & 1.217 & 51 & 24 & $\$ 650$ & $\$ 791.050$ \\
\hline TOTALES & & 35.531 & & & & $\$ 11.425 .120$ \\
\hline
\end{tabular}

Tabla 1. Relación de embarcaciones que operaron paseos por la bahía de Mazatlán con potencial de visita a RT para la observación de lobos marinos durante la temporada invernal 201 1-2012.

Fuente: Elaboración propia con datos de despacho de Capitanía de Puerto Mazatlán. 


\section{DISCUSIÓN}

La oferta turística de Mazatlán está basada en los atractivos naturales que ofrece la bahía, como playas, islas e islotes, siendo el turismo náutico una de las actividades con mayor demanda en el destino (Guzón-Zatarain et al., 2013).

\section{CARACTERIZACIÓN DE LA ACTIVIDAD TURÍSTICA}

Según los registros analizados, durante la temporada invernal 2011-2012, 14 catamaranes de pasajeros operaron desde las flotas del puerto principal. Sin embargo, la información provista por la Capitanía de Puerto para diciembre del 2012 indica que en los embarcaderos de esta zona ofrecen servicio cerca de 20 catamaranes con capacidades que varían entre los 45 y 444 pasajeros (Guzón-Zatarain et al., 2013). Por otra parte, es posible que el número de embarcaciones menores, obtenido de los registros (cuatro pangas), es significativamente menor al de la flota operativa real, ya que en los registros de la Dirección General de Marina Mercante y la Dirección de Registro y Programas existían, para el periodo 2003-2005, al menos 38 embarcaciones menores 0 pangas con permiso de turismo náutico operando desde diversas flotas del puerto principal, de las cuales 22 contaban también con permiso para transportación de pasajeros. Estas últimas pertenecen a la flota de pangas que operan como taxis acuáticos para cruzar pasajeros a la Isla de la Piedra, las cuales están representadas por al menos dos cooperativas: Unión de Propietarios de Canoas, Botes y Lanchas CROM y la Unión de Propietarios de Lanchas de la Isla de la Piedra A.C. y que operan desde al menos dos embarcaderos en la zona de
Playa Sur, las cuales también ofrecen los servicios de "paseo por la bahía" con énfasis en la observación de las "focas" (lobos marinos), donde éstas no aparecen en los despachos de Capitanía de Puerto para el periodo 2011-2012. Hay que añadir que recientemente la flota de pescadores artesanales de Playa Norte ha comenzado a ofrecer servicios turísticos catalogados simplemente como "paseos en lancha" los cuales incluyen la visita a la zona de Rocas Blancas y RT para la observación de lobos marinos, así como visitas a la Isla Venados.

Ante la gran demanda de servicios turísticos en la bahía de Mazatlán, sus islas y su zona marina adyacente, así como el tamaño y capacidad de las flotas turísticas, es evidente que los impactos al medio ambiente marino podrían ser significativos. Las actividades como paseos por la bahía, deportes acuáticos y visitas a las islas, los cuales son operados por catamaranes, embarcaciones menores y otro tipo de embarcaciones de recreo, tienen el potencial de ocasionar impactos que aún no han sido evaluados y que, por lo tanto, requiere de una atención prioritaria por parte del sector para lograr su adecuado manejo y regulación (Guzón-Zatarain et al., 2013).

Es evidente que existe mucho mayor control de las embarcaciones que operan desde Marina Mazatlán que las que operan desde el puerto principal. Esta posible subestimación del número de embarcaciones en la flota operativa también estaría subestimando el número de usuarios que contrataron servicios de turismo náutico durante la temporada invernal 2011-2012. Por otra parte, si se incluyen los usuarios que contrataron servicios para otros paseos como la Isla de la Piedra, que comúnmente visitan RT, más 
del $95 \%$ de los usuarios registrados estarían Venados. Esta cifra representa más del potencialmente visitando la RT para la doble de la capacidad de carga permitida observación de lobos marinos, lo cual se y refleja también poca eficiencia en la traduce a aproximadamente 40.000 capacidad de manejo de esta área natural personas. Esta cifra por sí sola no tiene protegida. Además, la capacidad de carga particular relevancia, ante el hecho de que estimada en el estudio de Flores-Campaña no existe a la fecha ningún estudio que (2011) está calculada con datos de visitas estime la "capacidad de carga", término que se refiere al número máximo de turistas que puede albergar un área de destino (O'Reilly, 1991) turística para la visitación de RT. No obstante, se han distinguido distintas categorías de capacidad de carga turística, siendo la capacidad de carga ecológica-medioambiental propuesta por Watson y Kopachevsky (1996) la más adecuada para este tipo de visitas: "el nivel de desarrollo turístico o actividad recreacional más allá del cual el medio ambiente que conocemos se degrada o se ve comprometido y, por lo tanto, afecta al aire, el suelo, la vegetación, la fauna, el agua, y todos los demás elementos físicos que componen un ecosistema". Actualmente este es uno de los aspectos más importantes a considerar para la configuración de un producto turístico. Los estudios de Flores-Campaña (2011) sobre la sustentabilidad de la actividad turística en las tres islas de Mazatlán, que junto con RT forman parte del APFF Islas del Golfo de California, Sinaloa, determinó que la capacidad de carga efectiva que se puede permitir en la playa y el sendero de la Isla Venados es de 1.361 personas y 29 visitas/día, respectivamente, considerando un escenario del $50 \%$ de eficiencia en la capacidad de manejo del área.

durante julio y agosto que es una temporada de una menor afluencia turística dominada en particular por turismo nacional. Siendo la temporada de invierno, entre noviembre y mayo, la de mayor afluencia turística, incluyendo gran parte del turismo internacional, podríamos esperar que la tasa de visitas a las islas sea mayor durante estos meses, resultando en un exceso aún mayor de la capacidad de carga establecida. Se requieren estudios que estimen la capacidad de carga turística real y coordinar esfuerzos con las instituciones responsables de su manejo (CONANP) para lograr mejores prácticas de turismo en el APFF Islas del Golfo de California, Sinaloa, incluyendo las islas Pájaros, Venados y Lobos, así como los islotes Dos Hermanos y Roca Tortuga, tanto para la observación de los lobos marinos como de otras especies de vida silvestre.

Considerando los registros analizados para lc Considerando los registros analizados para La estimación de los beneficios económicos las embarcaciones que visitan la Isla totales generados por servicios recreativos Venados $(n=4)$ (e.g. Kolonahe, Romance, en áreas naturales es un proceso complejo Konakai) se transportaron un total de 2.810 que requiere la integración de múltiples personas entre noviembre del 2011 y mayo variables. El método de "costo de viaje" fue del 2012, las cuales potencialmente una de las primeras aproximaciones a esta desembarcaron en la playa de Isla valoración e incluía los costos totales 
(transportación, hospedaje, alimentos y tarifas) en los que incurren los individuos para efectuar un viaje a un área natural y disfrutar de sus recursos (Clawson, 1959). Otros métodos incluyen la valoración contingente o disposición a pagar (DAP), en el cual a través de encuestas se solicita a las personas que expresen su disposición a pagar bajo un escenario hipotético, considerando una descripción del servicio ambiental (Randall, 1985). Este método es útil para determinar valores de uso y valores pasivos, pero ha sido más utilizado para estos últimos (Cárdenas-Torres, 2006).

De cualquier forma, la observación de lobos marinos en RT tiene un valor económico que va más allá del precio que se paga por su disfrute. Por lo que el "costo de viaje" representa solo una fracción del valor económico total de los lobos marinos como recurso y en el mejor de los casos las estimaciones obtenidas representan más bien la derrama económica generada por la actividad turística en torno al lobo marino de California en la bahía de Mazatlán. El beneficio económico neto total que genera un recurso natural es la suma de los beneficios netos generados por sus usos, así como los generados por su no uso. El valor de uso mide el valor de consumo o de uso directo de los recursos naturales tangibles, así como el valor de uso indirecto o de no-consumo. Los valores de uso directo pueden generarse por su aprovechamiento extractivo, tales como las ganancias netas de una pesquería, o bien por su aprovechamiento no-extractivo, tales como las ganancias generadas por turismo (Samonte-Tan et al., 2007). Los valores de uso indirecto son los beneficios funcionales que se derivan de los recursos tales como la protección de la zona costera y el hábitat para especies comerciales de peces o moluscos, mejor conocidos como servicios ambientales. A su vez, los valores de no-uso se dividen en: valor de opción loption value), valor de endoso (bequest value) y valor de existencia (existence value) (Cárdenas-Torres, 2006; Samonte-Tan, et al., 2007). Por ello, y dadas las importantes ganancias económicas que genera la actividad turística, es fundamental la realización de estudios que permitan estimar los beneficios económicos totales que representa el lobo marino de California, así como de las diferentes actividades productivas que hacen uso de los mismos, particularmente la pesca, el turismo y la industria portuaria.

Por otro lado, y de acuerdo con el Decreto (26-04-1991) del Área de Protección de Flora y Fauna (APFF) Islas del Golfo de California, las islas Lobos, Venados y Pájaros, así como los islotes Dos Hermanos y Roca Tortuga en la Bahía de Mazatlán se encuentran dentro de esta categoría de Área Natural Protegida (ANP) y, por lo tanto, es la CONANP la institución que tiene la obligación de manejar y administrar dichas áreas. De acuerdo con el reglamento en materia de cobro de derechos para el ingreso a las ANP del país, toda persona que desee ingresar a ellas debe pagar una cantidad específica. Para el caso de las islas de Mazatlán, la tarifa de pago de derechos es de \$27 pesos por persona. Si se considera a las 35.531 personas que contrataron servicios de turismo náutico, solamente durante la temporada invernal de noviembre del 2011 a mayo del 2012, como usuarios del APFF Islas del Golfo de California, el ingreso total sería de \$959.337 MXN Al no realizarse este cobro por parte de las autoridades correspondientes, este monto no ingresa a la Federación y, por lo tanto, no pueden ser invertidos en la aplicación de programas y acciones para la conservación y manejo del área, entre éstas la protección del lobo marino de California. 
Por último, cabe señalarse que la actividad ecoturística de observación de lobos marinos debería seguir las gestiones diseñadas en el plan de manejo de ANP, que menciona: i) las actividades de ecoturismo deben tener un bajo impacto ambiental y deben complementarse con instrucción ambiental y representar un beneficio directo para las comunidades locales; ii) las actividades deben utilizar únicamente las rutas de tránsito y las rutas de navegación existentes; iii) los visitantes no pueden recoger ningún elemento natural; y iv) la guía de turismo o el proveedor de servicios será responsable de cualquier daño causado al hábitat.

\section{CONCLUSIONES}

A través de esta investigación se demuestra que, al menos en 2012, casi 2 de cada 100 personas que visitaron el puerto de Mazatán (SECTUR, 2014) interaccionó para esta actividad ecoturística en con los lobos marinos de RT, especie específico, siguiendo criterios como los de considerada en la lista de protección de la Newsome y Rodger (2008).

NOM 059. El número de personas que visitó RT en esta temporada, que aumenta $\mathrm{Se}$ sugiere implementar actividades de anualmente (acorde con Toledo, 2020), educación para la conservación previas o sugiere que el nivel de perturbación de las durante el recorrido ecoturístico que condiciones naturales de los lobos marinos moldeen positivamente el comportamiento ante determinadas actividades turísticas, de los visitantes durante las actividades como el acercamiento de botes y lanchas a turísticas (González y Cubero, 2010) y la lobera, pudiera ser detrimental para la cerca de la fauna como el lobo marino en especie. Estudios previos del impacto RT en Mazatlán. Por otro lado, la mayoría ecoturístico en colonias de lobos marinos de los usuarios, turistas y locales, asimismo realizados en Australia (Orsini y Newsome, los operadores o guías turísticos poseen un 2005; Lovasz et al, 2008) y Ecuador escaso conocimiento del manejo y (Denkinger et al, 2014) sugieren acercamiento a estas especies, por lo que implementar recomendaciones de manejo, se sugiere invertir un porcentaje de las capacitación de guías turísticas y ganancias generadas por la actividad en educación para la conservación en el capacitar a los operadores en torno a la servicio ecoturístico a fin de garantizar la ecología de la especie y su ecosistema, así sustentabilidad de este tipo de turismo. Los como las interacciones humano-fauna que resultados de este estudio sugieren que un influyen en éstas. Se sugiere que estas 
capacitaciones sean diseñadas e implementadas de manera conjunta e incluyendo a las autoridades locales correspondientes, usuarios y prestadores de servicios.

\section{REFERENCIAS BIBLIOGRÁFICAS}

Aguiar, E. (2015). Observación participante: una introducción. Revista San Gregorio Edición Especial (1), 80-89. https://revista.sangregorio.edu.ec/index. php/REVISTASANGREGORIO/article/vie $w / 116 / 72$

Andersson, J. (2001). To Estimate Recreational Welfare Measures for International Tourism. Gothenburg University.

Angrosino, M. (2012). Etnografía y observación participante en investigación cualitativa. Ediciones Morata.

Aurioles-Gamboa, D., y Zavala-González, A. (1994). Algunos factores ecológicos que determinan la distribución y abundancia del lobo marino Zalophus californianus, en el Golfo de California. Ciencias Marinas, 20(4), 535-553. hitps://www.redalyc.org/pdf/480/48020405.pdf

Bechtold, C., y Parisi, R. (2020). Klemm Fellowship-Conservation in the Galapagos; Ecuador: Giant Tortoise \& Sea Lion Conservation in the Galapagos Islands. Presentación disponible en la web. Union College. https: / / digitalworks.union.edu/steinmetzsymposium/steinmetzthirty/oralpresentations/81/

Bringas, N., y Ojeda, L. (2003). El ecoturismo ¿una nueva modalidad del turismo de masas?. Economía, sociedad y territorio, 2(7), 373-404. hitps://doi.org/10.22136/est002000436

Camacho, R. (28 de junio de 2016). Lobos marinos: ¡Visitantes distinguidos!. Vámonos a Mazatlán. hitp://vamonosamazatlan.com.mx/lobos-marinos-visitantes-distinguidos/.

Cárdenas-Torres, A. (2006). Valoración económica de la actividad recreativa con tiburón ballena y su relación con la calidad del hábitat en Bahía de los Ángeles, Baja California. Tesis de Maestría en Oceanografía Costera. Universidad Autónoma de Baja California.

Chung, M., Dietz, T. y Liu, J. (2018). Global relationships between biodiversity and nature-based tourism in protected areas. Ecosystem Services, $34,11-23$.

Cifuentes-Lemus J., y Gaxiola-López, J. (2003). Atlas de los Ecosistemas de Sinaloa. El Colegio de Sinaloa.

Cisneros-Montemayor y Sumaila, A. (2010). A global estimate of benefits from ecosystem-based marine recreation: potential impacts and implications for management. Journal of Bioeconomics, 12(3), 245-268.

Clawson, M. (1959). Methods for measuring the demand for and the benefits for outdoor recreation. Resources for the future.

Comisión Intersecretarial para el Manejo Sustentable de Mares y Costas. (2012). Política Nacional de Mares y Costas. Gestión Integral de las Regiones más Dinámicas del País. 
Espinosa Martínez, R. (2014). Hacia un modelo de turismo competitivo y sustentable en Mazatlán y el sur de Sinaloa, Documento Síntesis Socios México/Centro de Colaboración Cívica. Costas y Comunidades (CONSELVA), Consejo para el Desarrollo de Sinaloa (CODESIN). DF. México.

Corrigan, C., Bingham, H., Shi, Y., Lewis, E., Chauvenet, A. y Kingston, N. (2018). Quantifying the contribution to biodiversity conservation of protected areas governed by indigenous peoples and local communities. Biological Conservation, 227, 403-412. https://doi.org/10.1016/i.biocon.2018. 09.007

Crofts, R. (2019). Linking geoconservation with biodiversity conservation in protected areas. International Journal of Geoheritage and Parks, 7(4), 211-217. https://doi.org/10.1016/j.ijgeop.2019.12.002

Das, M. y Chatteriee, B. (2015). Ecotourism: A panacea or a predicament?. Tourism Management Perspectives, 14, 3-16. https://doi.org/10.1016/i.tmp.2015.01.002

Denkinger, J., Quiroga, D., y Murillo, J. (2014). Assessing human-wildlife conflicts and benefits of Galapagos sea lions on San Cristobal Island, Galapagos. En The Galapagos Marine Reserve. Springer, C. (Ed.). Pp 285-305. https://link.springer.com/chapter/10.1007/978-3-319-02769-2_13

Díaz-Bravo, L., Torruco-García, U., Martínez-Hernández, M., y Varela-Ruiz, M. (2013). La entrevista, recurso flexible y dinámico. Investigación en educación médica, 2(7), 162-167.

Díaz-Maestre, B. (2017). Estado Actual del Garrod, B., y Wilson, J. C. (2003). Lobo Marino de California (Zalophus Cali- Conclusions. En Autores (Eds.) Marine fornianus) en Mazatlán, Sinaloa: Infor- ecotourism: issues and experiences. mación Base para el Manejo Responsable. Channel View Publications.
Tesis de Maestría. Universidad Autónoma de Sinaloa.

Díaz-Maestre, B., Guzón-Zatarain, O. Roldán-Clarà, B., y Grano-Maldonado, M. (2019). Un biomodelo turístico en el Pacífico mexicano: características biológicas y ambientales asociadas a la actividad sustentable del lobo marino Zalophus californianus (Lesson 1828). Biotempo, 16(1), $41-60$.

Díaz-Maestre, B., Guzón-Zatarain, ○. Mendieta-Vega, R., Roldán-Clarà, B., y Grano-Malonado, M. (2020). La influencia del turismo, las practices de gestion ecológica y la perspectiva social durante la observación de lobos marinos (Zalophus californianus) (Lesson 1828) en Mazatlán, México. Biotempo, 17(2).

Drum, A., y Moore, A. (2004). Volume I: An Introduction to Ecotourism Planning. En Singer, A. (Ed.). Ecotourism Development. A Manual for Conservation Planners and Managers. The Nature Conservancy.

Flores-Campaña, L. (2011). Modelo de manejo sustentable de los recursos naturales y la actividad turística de las 3 Islas de Mazatlán, Sinaloa, México. Tesis de Doctorado. Universidad de Guadalajara.

Gallagher, A. J., y Hammerschlag, N. (2011). Global shark currency: the distribution, frequency, and economic value of shark ecotourism. Current Issues in Tourism, 14/8), 797-812. https://doi.org/10.1080/13683500.2 011.585227 
Gobierno del Estado de Sinaloa. (1991). Decreto de Zona de Reserva Ecológica y Refugio de Aves Marinas y Migratorias y de Fauna y Flora Silvestres. Periódico Oficial El Estado de Sinaloa, (50).

González, F. Cubero-Pardo, P. (2010). Efecto de actividades turísticas sobre el comportamiento de fauna representativa de las Islas Galápagos, Ecuador. Latin American Journal of Aquatic Research 38(3), 493-500. hitps://www.scielo.cl/scieI o. ph p ? script=sci_arttex t\&pid=S07 18-560X2010000300013

Guzón-Zatarain, O. R., Rosales-Rodríguez, M. y Márquez-Farias, F. (2013). Estado de la sustentabilidad de la actividad turística en Mazatlán, Sinaloa. Ecosistemas Costeros Sustentables. Sinaloa.

Habibullah, M. S., Din, B. H., Chong, C. W., y Radam, A. (2016). Tourism and biodiversity loss: implications for business sustainability. Procedia Economics and Finance, 35, 166-172. https://doi.org/10.1016/S2212-5671/16/000 $21-6$

Instituto de Estadística en América Latinoa y el Caribe . (16 de septiembre de 2020). Principales sectores de actividad económica en Sinaloa. http://cuentame.inegi.org. mx/monografias/informacion/Sin/Economia/default.aspx?tema $=M$ $\mathrm{E \& e}=25$

Johnson, A. F., González, C., Townsel, A. y Cisnernos-Montemayor, A. M. (2019). Marine ecotourism in the Gulf of California and the Baja California Peninsula: Research trends and information gaps. Scientia Marina, 83(2), 177-185. https://www.torrossa.com/en/resources/ an $/ 4574118 \#$ page $=99$
Kido Cruz, M. T., y Cuéllar, M. (2010). Impacto económico por el pago de pasajeros de cruceros para la conservación de recursos naturales: el caso de Bahías de Huatulco, México. Economía, sociedad y territorio, 10(32), 81-97. http: / / www.scielo.org.mx/scielo.php?pid $=$ S 1 405-84212010000100004\&script=s ci_arttext

King, J.E. (1983). Seals of the World. Comstock Publishing Assoc. Comell University Press, N.Y., 240 pp.

López-Espinosa, R. (2002). Evaluating ecotourism in natural protected areas of $\mathrm{La}$ Paz Bay, Baja California Sur, México: ecotourism or nature-based tourism? Biodiversity \& Conservation, $17(9), 1539-1550$.

Lovasz, T., Croft, D., y Banks, P. (2008). Establishing tourism guidelines for viewing Australian Sea Lions Neophoca cinerea at Seal Bay Conservation Park, South Australia. En Lunney, D., Munn A., Meikle W., (Eds). Too Close for Comfort: Contentious Issues in Human-Wildlife Encounters lpp. 225-2321. Royal Zoological society of New South Wales.

Martín, F. (2011). La encuesta: una perspectiva general metodológica. Centro de Investigaciones Sociológicas.

Mathis, A., y Rose, J. (2016). Balancing tourism, conservation, and development: a political ecology of ecotourism on the Galapagos Islands. Journal of Ecotourism, 15111, 64-77. https://doi.org/10.1080/14724049.2015.11 31283

Miller, M. L. (1993). The rise of coastal and marine tourism. Ocean \& Coastal Management, 2931, 181-199. https://doi.org/10.1016/0964-5691/93)90066-8 
Nava Z., y lbarra, G. (2014). Innovación territorial para la competitividad de los espacios turísticos. El caso de Mazatlán, Sinaloa. En Ibarra G. y Ruelas, A. L., (Coords.), Entre lo global y lo local. Ciencias sociales en Sinaloa, México, (pp. 13-48). Juan Pablos Editor UAS.

Newsome, D., y Rodger, K. (2008). Impacts of tourism on pinnipeds and implications for tourism management. En Higham, J., y Lück, M. (Eds.), Marine wildlife and tourism management: Insights from the natural and social sciences (pp. 182-205). CAB International.

O’Reilly, Ainsley M. (1991) Tourism carrying capacity. En: Medlink, S. (ed.) Managing Tourism. Oxford: Butterworth-Heinemann. 344pp.

Orsini, J., Newsome, D. (2005). Human perceptions of hauled out Australian sea lions (Neophoca cinerea) and implications for management: a case study from Carnac Island, Western Australia. Tourism in Marine Environments, 2(1), 129-132. https://doi.org/10.3727/154427305774865 769

Peterson, R.S., y Bartholomew, G.A. (1967). Natural History and behavior of the California sea lion. The American Society of Mammalogist. https://www. biodiversitylibrary.org/item/87697\#page/8/mode/2up

Randall, A. (1985). Economía de los Recursos Naturales y Política Ambiental. Limusa. Rodríguez, M. B., y Valiente, G. C. (2019). El desarrollo turístico en Mazatlán, México: evaluación de la sostenibilidad por medio de indicadores. Cuadernos de Turismo, (43), 87-213. doi.org/10.6018/turismo.43.08
Samonte-Tan, G., White, A., Tercero, M., Diviva, J., Tabara E., y Caballes, C. (2007). Economic Valuation of Coastal and Marine Resources: Bohol Marine Triangle, Philippines. Coastal Management, (35), 319-338. hitps://doi.org/10.1080/08920750601 169634

Sampieri-Hernández R., Fernández C., y Baptista, P. (2010). Metodología de la investigación. McGraw-Hill.

Secretaría de Turismo. (2014). Agenda de competitividad del destino turístico Mazatlán. http://www.sectur.gob.mx/wp-content/uploads/2015/02/PDF-Mazatlan.pdf

Secretaría de Turismo. (2007). Elementos para Evaluar el Impacto Económico, Social y Ambiental del Turismo de Naturaleza en México. https://manuelmiroglio.files.wordpress.com/2011/06/estudioturismo_de_naturaleza.pdf

Secretaría de Medio Ambiente, Recursos Naturales y Pesca. (2000). Acuerdo que tiene por objeto dotar con una categoría acorde con la legislación vigente a las superficies que fueron objeto de diversas declaratorias de áreas naturales protegidas emitidas por el Ejecutivo Federal. Gaceta Ecológica, (54), 83-87, [fecha de consulta 7 de noviembre de 2021]. https://www.redalyc.org/pdf/539/53905407.pdf

Secretaría del Medio Ambiente y Recursos Naturales. (2010). Norma Oficial Mexicana NOM-059-SEMARNAT-2010. Protección ambiental-Especies nativas de México de flora y fauna silvestres-Categorías de riesgo y especificaciones para su inclusión, exclusión o cambio-Lista de especies en riesgo. Diario Oficial de la Federación, 30-12-2010. 
Sierra, F. (1998). Función y sentido de la y estrategias en la investigación cualitativa, entrevista cualitativa en investigación social. 18-34.

En J. Galindo-Cáceres (Ed.), Técnicas de investigación en sociedad, cultura y comunicación (pp. 277-354). Pearson Addison Wesley.

Watson, L., y Kopachevsky, J. (1996). Tourist Carrying Capacity: A critical look at the discursive dimension. Progress in Tourism and Hospitality research, 2(2), 169-179.

Toledo, M. (09 de enero del 2020). Llegan más de 4.7 millones de turistas a Sinaloa en 2019. El Sol de Mazatlán. https://www. elsoldemazatlan.com.mx/local/llegan-mas-de-4.7-millones-de-turistas-a-sinaloa -en-2019-4680579.html https://doi.org/10.1002/pth.6070020 205

Wondirad, A. (2019). Does ecotourism contribute to sustainable destination development, or is it just a marketing hoax? Analyzing twenty-five years contested journey of ecotourTrindade, V. A. (2016). Entrevistando en investigación cualitativa y los imprevistos en el trabajo de campo: de la entrevista semiestructurada a la entrevista no estructurada. ism through a meta-analysis of tourism journal publications. Asia Pacific Journal of Tourism Research, 24(111), 1047-1065. https://P, Schettini y I.Cortazzo (Coords.), Técnicas 557 